OPEN ACCESS

Edited by:

Kang-le Lu,

Jimei University, China

Reviewed by:

Yun-Zhang Sun,

Jimei University, China

Rui Jia,

Chinese Academy of Fishery

Sciences, China

Jin Niu,

Sun Yat-sen University, China

*Correspondence:

Yanjiao Zhang

yanjiaozhang@ouc.edu.cn

Specialty section:

This article was submitted to Marine Fisheries, Aquaculture and

Living Resources,

a section of the journal

Frontiers in Marine Science

Received: 17 July 2020 Accepted: 06 November 2020 Published: 27 November 2020

Citation:

Dai J, Ou W, Yu G, Ai Q, Zhang W,

Mai $K$ and Zhang $Y$ (2020) The Antimicrobial Peptide Cecropin AD

Supplement Alleviated Soybean Meal-Induced Intestinal Inflammation,

Barrier Damage, and Microbial

Dysbiosis in Juvenile Turbot,

Scophthalmus maximus.

Front. Mar. Sci. 7:584482

doi: 10.3389/fmars.2020.584482

\section{The Antimicrobial Peptide Cecropin AD Supplement Alleviated Soybean Meal-Induced Intestinal Inflammation, Barrier Damage, and Microbial Dysbiosis in Juvenile Turbot, Scophthalmus maximus}

\author{
Jihong Dai ${ }^{1}$, Weihao Ou ${ }^{1}$, Guijuan $\mathrm{Yu}^{1}$, Qinghui $\mathrm{Ai}^{1}$, Wenbing Zhang ${ }^{1}$, Kangsen Mai ${ }^{1,2}$ and \\ Yanjiao Zhang ${ }^{1,2 *}$ \\ ${ }^{1}$ The Key Laboratory of Aquaculture Nutrition and Feed (Ministry of Agriculture) \& The Key Laboratory of Mariculture (Ministry \\ of Education), Ocean University of China, Qingdao, China, ${ }^{2}$ Laboratory for Marine Fisheries Science and Food Production \\ Processes, Qingdao National Laboratory for Marine Science and Technology, Qingdao, China
}

This study aimed to investigate the benefit of dietary cecropin AD (CAD) on the intestinal health of turbot (Scophthalmus maximus) fed diets with a high level of soybean meal. A 12-week feeding trial was conducted with four isonitrogenous and isolipidic diets: a fishmeal-based diet (FM), a diet replacing $40 \%$ fish meal protein of FM diet with soybean meal protein (SBM), and the SBM diet supplemented with $0.5 \mathrm{~g} / \mathrm{kg}(\mathrm{C} 1)$ and $1.0 \mathrm{~g} / \mathrm{kg}$ (C2) CAD, respectively. The results of histology of distal intestine (DI) showed that turbots fed the SBM diet exhibited distinct symptoms of enteritis. However, fish fed diets with CAD supplementation kept the normal appearance of the DI which was similar to that in FM group. Compared with the SBM group, diet with CAD supplementation significantly decreased the gene expression of intestinal pro-inflammatory cytokines tumor necrosis factor- $\alpha$ (tnf- $\alpha$ ), interleukin-1 $\beta$ (il-1 $\beta$ ), interferon-gamma (ifn- $\gamma$ ), and nuclear factor-kappa B p65 (nf-кb p65), while up-regulated the gene expression of intestinal tight junction proteins claudin-3, claudin-4, occludin, and zonula occludens-1 (zo-1). Besides, diet C1 shaped the intestinal microbiota profile toward an anti-inflammatory phenotype represented by the increased abundance of Blutia, Firmicutes/Bacteroides ratio, and decreased Prevotellaceae. In conclusion, dietary CAD could positively modulate the intestinal health of turbot from the impairment induced by soybean meal, which expands its application to help fish better adapt to the increasing plant protein level in aquafeed.

Keywords: cecropin AD, intestinal inflammation, intestinal tight junction, intestinal microbiota, turbot 


\section{INTRODUCTION}

Soybean meal has been widely used in aquafeed to solve the contradiction between the soaring requirement and the lacking supply of fishmeal. However, dietary inclusion of soybean meal often leads to soybean meal-induced enteritis (SBMIE) in marine fish, resulting in inferior health conditions and poor growth performance (Baeverfjord and Krogdahl, 1996; Krogdahl et al., 2003; Chen et al., 2018a; Liu et al., 2018). The SBMIE in fish is commonly featured by overexpressed pro-inflammatory cytokines, disruption of barrier function, microbial dysbiosis, as well as evident pathological changes (Baeverfjord and Krogdahl, 1996; Mansfield et al., 2010; Reveco et al., 2014; Zhou et al., 2018; Liu et al., 2019). As an indispensable component of intestinal barrier, tight junction (TJ) complex in terms of morphology and gene expression analysis has been extensively investigated in fish suffering from SBMIE (Merrifield et al., 2009; Liu et al., 2019; Zhao et al., 2019), as a healthy TJ plays pivotal roles in resisting the invasion of harmful substances (DeMeo et al., 2002) and preventing intestinal inflammation from prolongation and aggravation (Su et al., 2013). Meanwhile, a healthy gut also depends on the balance of microbial ecosystem, which acts as a defensive barrier combined with the role in aiding digestive function and development of intestine (Luis Balcázar et al., 2006). Given that intestinal microbial composition and barrier function can be effectively modified by dietary ingredients, and their significance in maintenance of intestinal homeostasis (Clements et al., 2014; Li et al., 2017; Zhou et al., 2018), many nutritional strategies such as functional amino acid/protein, probiotics, prebiotics, and organic acid have been exploited to relief fish SBMIE (Sealey et al., 2009; Refstie et al., 2010; Ulloa et al., 2016; Chen et al., 2018b; Liu et al., 2018; Zhao et al., 2019).

Antimicrobial peptides (AMP) are important components of innate immune defense with broad-spectrum antibiotic activity and immunomodulatory activities (Reddy et al., 2004; Hancock et al., 2016), hence various kinds of AMPs have been investigated to improve immune response and enhance disease resistance in fish (Zhou et al., 2008; Dong et al., 2015; Lin et al., 2015; Su et al., 2019; Li et al., 2020). Cecropin AD (CAD) is an artificial cationic amphipathic AMP possessing great potential for commercial application as a functional additive due to its relatively stable bioactivity, broad-spectrum antimicrobial property, and easily acquisition as a well-designed Bacillus subtilis expression system has been established (Chen et al., 2009). Studies have shed light on the effectiveness of CAD in reducing intestinal aerobic bacteria and potential pathogens in piglets and broilers (Wen and He, 2012; Wu et al., 2012). Moreover, some cecropin family peptides exert great anti-inflammatory activity in mammal immune cells (Kim et al., 2011; Lee et al., 2015;

\footnotetext{
Abbreviations: CAD, cecropin AD; FE, feed efficiency; FI, feed intake; SGR, specific growth rate; FM, fishmeal; SBM, soybean meal; $\operatorname{tg} f-\beta$, transforming growth factor- $\beta$; $t n f-\alpha$, tumor necrosis factor- $\alpha$; $i l-1 \beta$, interleukin- $1 \beta$; $n f-\kappa \mathrm{b}$ p 65 , nuclear factor-kappa B p65; zo-1, zonula occludens-1; ifn- $\gamma$, interferon-gamma; il-8, interleukin-8; il-22, interleukin-22; SBMIE, soybean meal-induced enteritis; TJ, tight junction; AMP, antimicrobial peptide; OTU, operational taxonomic unit; PCoA, principle coordinate analysis; DI, distal intestine; LEfSe, linear discriminant analysis effect size.
}

Wu et al., 2015) and promote the expression of TJ proteins in porcine jejunum epithelial cells (Zhai et al., 2018). More recently, our previous study proved that dietary CAD reduced the abundance of intestinal Bacteroides while increased Lactococcus lactis, highlighting its role as an intestinal immunoenhancer in turbot (Scophthalmus maximus) fed a diet whose composition resembled commercial formulation (Dai et al., 2020). Therefore, the present study was designed to clarify the beneficial effects of dietary CAD to protect the intestine health from the soybean meal-induced intestinal inflammation, barrier damage, and microbial dysbiosis in turbot, which is an extensively cultured marine carnivorous fish in the world.

\section{MATERIALS AND METHODS}

\section{Ethical Clarification}

All sampling protocols, as well as fish rearing practices, were reviewed and approved by the Animal Care and Use Committee of Ocean University of China.

\section{Experimental Diets}

A total of four isonitrogenous and isolipidic experimental diets were formulated to contain $52 \%$ crude protein and $12 \%$ crude lipid: fishmeal-based diet (FM); FM with $40 \%$ fish meal protein replaced by soybean meal protein (SBM); SBM +0.5 $\mathrm{g} / \mathrm{kg}$ CAD (C1) and SBM+1.0 g/kg CAD (C2) (Table 1). The $\mathrm{CAD}$ used in this study was developed by the National Feed Technology Development Research Center and purchased from Peking Sinagri Yin Thai Bio-Technology (Beijing, China). The amino acid sequence composition of CAD is KWKLFKKIEKVGQRVRDAVISAGPAVATVAQATALAK with biomass of $3.8 \mathrm{KDa}$. A Bacillus subtilis expression system was used for the mass-production of CAD. Briefly, the CAD gene was fused with a small ubiquitin-like modifier gene and a signal peptide of SacB. B. subtilis expression system was constructed based on $B$. subtilis cells genetically modified by introducing an operon comprising a promoter, the SUMO protease gene, and a signal peptide of amyQ (Chen et al., 2009). The process of diet making followed our previous study (Dai et al., 2020).

\section{Fish Husbandry}

Apparently healthy juvenile turbots were obtained from a commercial fish farm in Penglai (Shandong, China). Fish were fed the FM diet for 14 days to adapt to the experimental conditions before the initiation of the feeding trial. At the start of the experiment, 4 groups of turbot with similar sizes (initial mean weight $8.47 \pm 0.04 \mathrm{~g}$ ) were randomly distributed to 12 fiberglass tanks (200L, 30 fish/tank). Each diet was randomly fed to three replicate groups of turbot to apparent satiation twice daily (08:00 and 17:00). Uneaten pellets were collected by siphonage, then dried and weighed for the calculation of feed intake. The tanks were supplied with seawater collected from the adjacent coast after sedimentation and sand filtration, with water flow rate at about $3 \mathrm{~L} / \mathrm{min}$; a temperature between $15^{\circ} \mathrm{C}$ and $17^{\circ} \mathrm{C}$; salinity, $35 \%$; $\mathrm{pH}$ ranged from 7.5 to 8.0; dissolved oxygen over $6 \mathrm{mg} \mathrm{L}^{-1}$. The fish were kept into a flow-through water system supplied with continuous aeration. 
TABLE 1 | Formulations and chemical analyses of the experimental diets (\% DM).

\begin{tabular}{lcccc}
\hline Ingredient (\%) & FM & SBM & C1 & C2 \\
\hline Fishmeal $^{\mathrm{a}}$ & 66 & 40 & 40 & 40 \\
Soybean meal $^{\mid \mathrm{a}}$ & 0 & 35 & 35 & 35 \\
Wheat meal $^{\mathrm{a}}$ & 26 & 15.6 & 15.55 & 15.5 \\
Fish oil $_{\text {Soy lecithin }}$ & 5 & 6.4 & 6.4 & 6.4 \\
Monocalcium phosphate $_{\text {Choline chloride }}$ & 1 & 1 & 1 & 1 \\
Vitamin and Mineral premix $^{\mathrm{b}}$ & 0.5 & 0.5 & 0.5 & 0.5 \\
Calcium propionate & 0.3 & 0.3 & 0.3 & 0.3 \\
Ethoxyquin & 0.05 & 0.05 & 0.05 & 0.05 \\
Cecropin AD & 0.05 & 0.05 & 0.05 & 0.05 \\
$\mathrm{Y}_{2} \mathrm{O}_{3}$ & 0 & 0 & 0.05 & 0.1 \\
Chemical analysis & 0.1 & 0.1 & 0.1 & 0.1 \\
Crude protein (CP) & & & & \\
Crude lipid (CL) & 52.42 & 51.83 & 51.62 & 51.21 \\
\end{tabular}

${ }^{a}$ Fishmeal (DM, \%): CP 72.47, CL 9.00; Soybean meal (DM, \%): CP 54.54, CL 1.92; wheat meal (DM, \%): CP 17.68, CL 2.05.

${ }^{b}$ Vitamin and Mineral premix ( $m g \mathrm{~kg}-1$ ): retinyl acetate, 150; vitamin D3, 3.75; $D L-\alpha-$ tocopherol acetate, 4,000; vitamin K3, 500; thiamin, 500; riboflavin, 800; pyridoxine hydrochloride, 600; vitamin B12, 2; L-ascorbyl-2-monophosphate-Na, 10,000; calcium pantothenate, 2,000; nicotinic acid, 3,000; inositol, 10,000; biotin, 5; folic acid, 170, moisture < 10\%; Fe, 12,000; Zn, 6,000; $\mathrm{Cu}, 400 ; \mathrm{Mn}, 3,000 ; \mathrm{I}, 100$; $\mathrm{Co}$, 10; Se, 10; Mg, 15,000.

cPurchased from Peking Sinagri Yin Thai Bio-Technology (Beijing China).

\section{Sample Collection}

On the day 84 , all the fish were fasted for $24 \mathrm{~h}$ for the measuring of biomass in each tank. On the day 85, all turbots were fed $5 \mathrm{~h}$ previous to sampling. All turbots were fed $5 \mathrm{~h}$ previous to sampling to ensure the intestine was filled with contents. The contents were collected but not for use in the present study. Distal intestine (DI) of 3 fish per tank were dissected and rinsed with phosphate-buffered saline (PBS) to get rid of the contents before immersing in liquid nitrogen and storing at $-80^{\circ} \mathrm{C}$ for gene expression analysis. For the analysis of intestinal microflora, the abdomen surface of fish was cleansed with $70 \%$ ethanol. Afterward, the hindguts of other 3 fish each tank were collected with a sterile dissecting instrument near an alcohol lamp. The digesta samples were collected aseptically by squeezing the contents out of the DI into sterile containers and transferred into storage at $-80^{\circ} \mathrm{C}$. Then the remaining DI tissues were rinsed 3 times softly for $1 \mathrm{~min}$ in PBS to remove the contents before transferred to $2 \mathrm{~mL}$ sterile Axygen tubes and immediately frozen in liquid nitrogen. On the day 86 , Three fish per tank were randomly picked for the analysis of proximate composition. For the intestinal histological examination, short segments (about $1 \mathrm{~cm}$ in length) of DI from other 3 randomly selected fish were dissected and fixed in the $4 \%$ paraformaldehyde (Solarbio, China).

\section{Chemical Analysis}

The proximate composition of feed ingredients, diets and fish body were determined according to the standard methods
(AOAC, 1995). Dry matter was measured by drying samples at $105^{\circ} \mathrm{C}$ to a constant weight; crude protein by the Kjeldahl method (2300-Autoanalyzer, FOSS Tecator, Höganäs, Sweden); crude lipid by the Soxhlet method (B-811, BUCHI, Flawil, Switzerland); ash by combustion to a constant weight at $550^{\circ} \mathrm{C}$.

\section{Histological Analysis}

After fixation in the $4 \%$ paraformaldehyde for $24 \mathrm{~h}$, DI samples were dehydrated in ethanol, equilibrated in xylene, and embedded in paraffin according to standard histological techniques. Then the sections of $7 \mu \mathrm{m}$ were cut with a rotary microtome (Lecia Jung RM 2016, Germany) and stained with the hematoxylin-eosin method. The examination was conducted with a light microscope (DP 72, Olympus, Tokyo, Japan) equipped with a camera (E 600, Nikon, Tokyo, Japan) and an image acquisition software (CellSens Standard, Olympus, Tokyo, Japan) following the criteria suggested by krogdahl (Krogdahl et al., 2003): length and fusion of mucosal folds, cellular infiltration, and width of the lamina propria and submucosa, enterocyte vacuolization, and nucleus position within the enterocytes. The perimeter ratio (arbitrary units AU) between the internal and external perimeter was determined using the Image-Pro Plus ${ }^{\circledR}$ software (Media Cybernetics, Silver Spring, $\mathrm{MD}$, United States) according to the method of Dimitroglou (Dimitroglou et al., 2009), a schematic drawing showing the parts served to calculate the intestinal PR value was described in our previous study (Dai et al., 2018). A higher PR value indicates a larger absorptive surface area resulting from increased length of villi height and/or a number of mucosal folding.

\section{Quantitative RT-PCR Analysis}

Total RNA was extracted from DI using Trizol reagent (Takara, Beijing) and electrophoresed on a 1.2\% agarose gel to evaluate the integrity, with RNA concentration assessed using NanoDrop ${ }^{\circledR}$ ND-1000 spectrophotometer (Thermo Scientific, Waltham, MA, United States). The first cDNA strand was synthesized by reversely transcribing purified $1 \mu \mathrm{g}$ total RNA using PrimeScript RT reagent Kit with gDNA Eraser (RR047A; Takara Biotech) according to the manufacturer's indication. The real-time qPCR (rt-qPCR) assays were performed in a total volume of $25 \mu \mathrm{L}$ consisted of $1 \mu \mathrm{L}$ of $200 \mathrm{ng} / \mu \mathrm{L}$ complementary cDNA product, 1 $\mu \mathrm{L}$ of each specific primer $(10 \mu \mathrm{M}), 12.5 \mu \mathrm{L}$ of TYBR Green PCR Master Mix [c ${ }^{\circledR}$ Premix Ex Taq ${ }^{\mathrm{TM}}$ (Tli RNase H Plus)] (Takara, Japan), $9.5 \mu \mathrm{L}$ of diethylpyrocarbonate-treated water (SigmaAldrich). The reaction conditions of $\mathrm{qPCR}$ amplification were employed as following conditions: $95^{\circ} \mathrm{C}$ for $2 \mathrm{~min}$ and then 40 cycles of $95^{\circ} \mathrm{C}$ for $10 \mathrm{~s}, 60^{\circ} \mathrm{C}$ for $20 \mathrm{~s}$, and $72^{\circ} \mathrm{C}$ for $20 \mathrm{~s}$. A melting curve analysis was performed after each amplification phase to confirm that any product detected was specific to the desired amplicon. Fold changes were calculated after normalization to reference gene $\beta$-actin using relative quantitative method $\left(2^{-\Delta \Delta C T}\right)$. Primer sequences are listed in Table 2 .

\section{Intestinal Microbiota Profiling}

Total bacteria DNA was extracted from the DI mucosa layer of one fish on a super clean bench around alcohol flame using a QiAamp DNA stool Mini Kit (Qiagen, Germany). 16S rRNA 
TABLE 2 | Primers used for real-time PCR analysis.

\begin{tabular}{|c|c|c|c|}
\hline Target gene & Forward primer & Reverse primer & Genebank No. \\
\hline claudin-3 & GCCAGATGCAGTGTAAGGTC & CCGTCCAGGAGACAGGGAT & KU238180.1 \\
\hline claudin-4 & ATGTGGAGTGTGTCGGCTT & AGACCTTGCACTGCATCTG & MF370857 \\
\hline occludin & ACTGGCATTCTTCATCGC & GGTACAGATTCTGGCACATC & KU238182.1 \\
\hline tricellulin & GCCTACATCCACAAAGACAACG & TCATTCCCAGCACTAATACAATCAC & KU238183.1 \\
\hline$i l-1 \beta$ & СGCTTCCCCAACTGGTACAT & ACCTTCCACTTTGGGTCGTC & AJ295836.2 \\
\hline $\operatorname{tgf}-\beta$ & TCAGCATTCCAGATGTAGGTG & GGAGAGTGGCTTCAGTIITC & KU238187.1 \\
\hline$i f n-\gamma$ & GCTITCCCGATCATCTTCTG & GGTTTCCCAGATTCCCATTC & DQ400686.1 \\
\hline$i l-8$ & GGCAGACCCCTTGAAGAATA & TGGTGAACCCTTCCCATTAT & HQ872499 \\
\hline il-22 & GCTGCAGCGTGACTCACTA & CTGCAGGTACGTGAAGAGGA & JQ349070.1 \\
\hline
\end{tabular}

TABLE 3 | Effects of dietary supplementation of CAD on growth performance and feed utilization of turbot ${ }^{1}$.

\begin{tabular}{|c|c|c|c|c|}
\hline & FM & SBM & C1 & C2 \\
\hline Weight gain (\%) & $369.81 \pm 7.56^{c}$ & $269.78 \pm 14.09^{a}$ & $347.37 \pm 12.93^{b c}$ & $300.86 \pm 18.34^{a b}$ \\
\hline $\operatorname{SGR}^{2}\left(\%\right.$ day $\left.^{-1}\right)$ & $1.84 \pm 0.02^{b}$ & $1.56 \pm 0.04^{a}$ & $1.78 \pm 0.03^{b}$ & $1.65 \pm 0.05^{\mathrm{ab}}$ \\
\hline $\mathrm{FE}^{3}$ & $1.14 \pm 0.02^{b}$ & $0.93 \pm 0.03^{a}$ & $1.03 \pm 0.01^{\mathrm{ab}}$ & $0.93 \pm 0.03^{a}$ \\
\hline $\mathrm{Fl}^{4}\left(\%\right.$ day $\left.^{-1}\right)$ & $1.36 \pm 0.02^{a}$ & $1.47 \pm 0.02^{a b}$ & $1.47 \pm 0.01^{\mathrm{ab}}$ & $1.54 \pm 0.03^{b}$ \\
\hline
\end{tabular}

${ }^{1}$ Values represent means of 3 replicate tanks.

${ }^{2} S G R$, specific growth rate.

${ }^{3} F E$, feed efficiency.

${ }^{4} \mathrm{Fl}$, feed intake.

Different superscript letters within a row denote significant differences $(P<0.05)$.

TABLE 4 | Effects of dietary supplementation of CAD on whole-body composition of turbot ${ }^{a}$.

\begin{tabular}{|c|c|c|c|c|}
\hline & FM & SBM & C1 & C2 \\
\hline Moisture (\%) & $76.29 \pm 0.10$ & $75.76 \pm 0.09$ & $76.03 \pm 0.16$ & $76.18 \pm 0.43$ \\
\hline Crude protein (\%) & $68.91 \pm 0.54$ & $67.00 \pm 0.79$ & $68.22 \pm 0.29$ & $68.08 \pm 0.29$ \\
\hline Crude lipid (\%) & $3.58 \pm 0.11$ & $3.49 \pm 0.34$ & $3.66 \pm 0.14$ & $3.65 \pm 0.19$ \\
\hline Ash (\%) & $4.06 \pm 0.06$ & $4.47 \pm 0.16$ & $4.07 \pm 0.12$ & $4.03 \pm 0.04$ \\
\hline
\end{tabular}

avalues represent means of 3 replicate tanks. The approximate composition is dry weight based.

gene amplicon sequencing libraries of the V4 hypervariable region were prepared with quality checked on the Qubit@ 2.0 Fluorometer (Thermo Scientific), and pools of amplicons were sequenced at Novogene Technology Co., Ltd. (Tianjin, China) on an Ion S5TM XL platform. After data filtration and chimera removal, clean reads were then imported into Uparse software (Uparse $\mathrm{v} 7,0,1001$ ). The reads were then clustered to operational taxonomic units (OTUs) based on $97 \%$ sequence similarity cut off value. Alpha diversity including Shannon and observed species was calculated with QIIME (v1.7.0) and displayed with $\mathrm{R}$ software (v 2.15.3). Principal Coordinate Analysis (PCoA) displayed by WGCNA package, stat packages, and ggplot2 package in $\mathrm{R}$ software (Version 2.15.3) was performed to get principal coordinates and visualize from complex, multidimensional data. Linear discriminant analysis effect size (LEfSe) analysis was performed on the bacterial taxa relative abundance values to determine the presence of bacterial taxa with statistically significant changes in abundance in the intestinal microbiome from different treatment groups.

\section{Calculations and Statistical Analysis}

The following variables were calculated:

Weight gain $(\%)=100 \times($ final weight - initial weight $) /$ initial weight.

Specific growth rate $($ SGR, $\%$ day- 1$)=100 \times($ Ln final weightLn initial weight)/days.

Feed efficiency $(\mathrm{FE})=($ final weight - initial weight $) /$ feed consumed. 


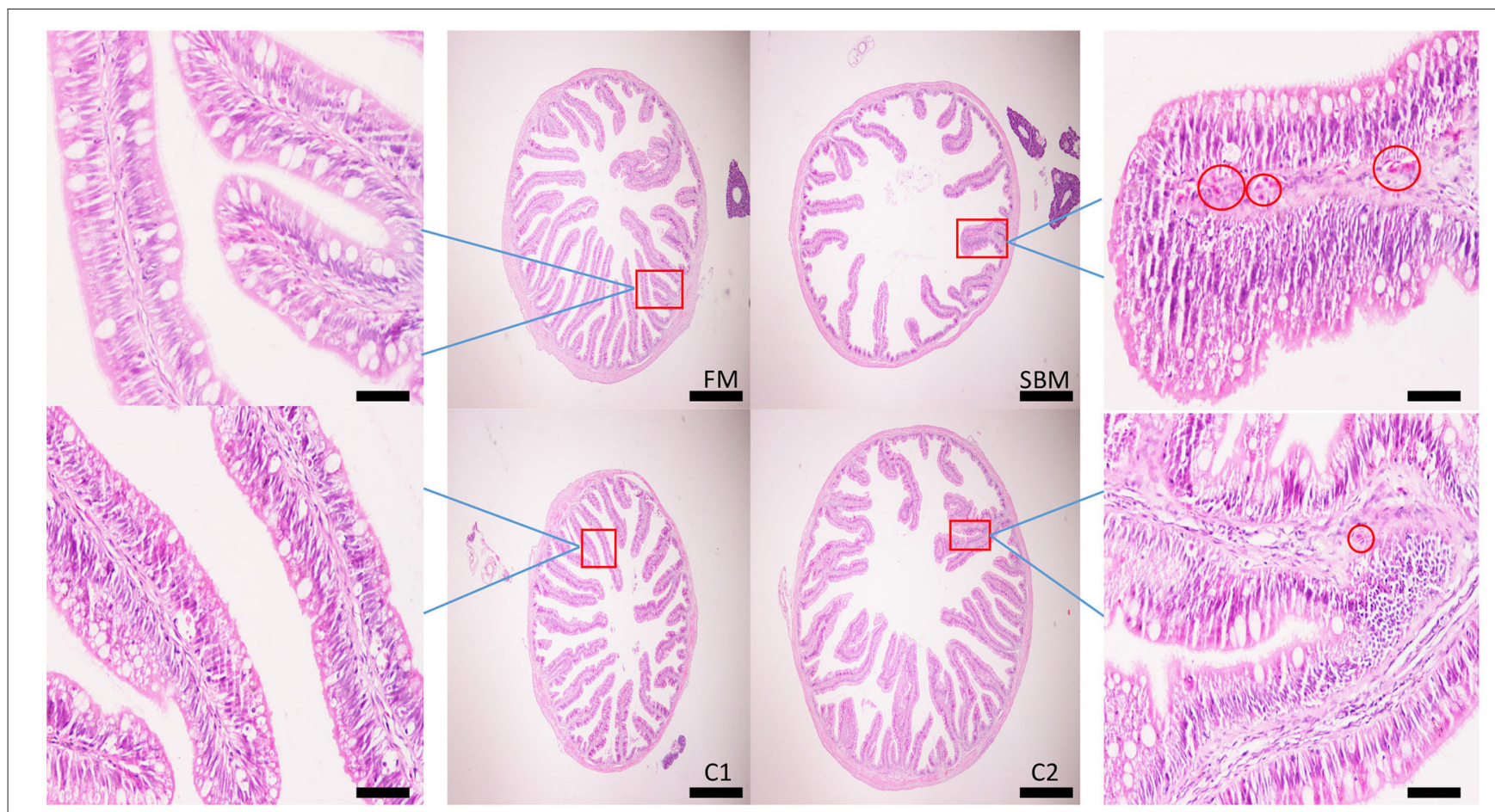

FIGURE 1 | Effects of dietary supplementation of CAD on morphology in the distal intestine (DI) of turbot. Scale bar of images at low power, $200 \mu \mathrm{m}$; Scale bar of enlarged images, $50 \mu \mathrm{m}$. The red circle denotes the dilation of the lamina propria with the infiltration of eosinophilic granulocyte.

Feed intake $($ FI, \% day- 1$)=100 \times$ feed intake $/[$ (initial weight + final weight)/2]/days.

SPSS version 22.0 for OS $\mathrm{X}$ was used for statistics analysis. Data except for microbiota analysis were subjected to homogeneity test before one-way analysis of variance (ANOVA). Differences between means were determined using Tukey's multiple-comparison test. Significance of results was determined when $P<0.05$, and the results are presented as means \pm SEM. Comparisons of 2 means were performed by using two-tail Student's $t$-test.

\section{RESULT}

\section{Growth Performance and Body Composition}

After the 12-week feeding trial, significantly enhanced weight gain and specific growth rate (SGR) were observed in FM and $\mathrm{C} 1$ groups compared with the SBM group $(P<0.05)$. The feed efficiency (FE) in fish fed diet $\mathrm{C} 1$ was higher than that in fish fed diet SBM, showing no significant difference with the FM group $(P>0.05)$ (Table 3). Whole-body composition was not affected by CAD supplementation as no treatment-related difference of crude protein, lipid, moisture, and ash were observed among all diet groups (Table 4).

\section{Intestinal Morphology}

Dietary CAD improved the villi morphology of the distal intestine (DI) both in quantity and the quality (Figure 1). The increased intestinal perimeter ratio was observed in the

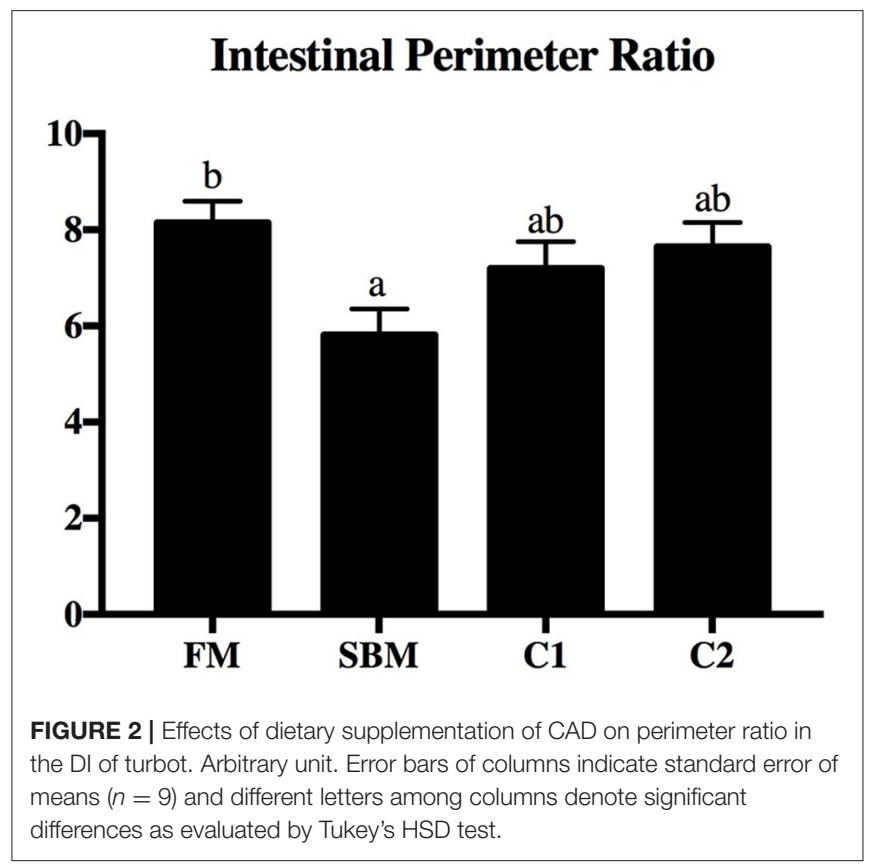

CAD-supplemented group compared with the SBM group (Figure 2). From the zoomed-in images, the broaden of lamina propia and infiltration of eosinophilic granulocyte were clearly observed in the SBM group, while the lamina propia slightly widened and fewer eosinophilic granulocytes accumulated in the $\mathrm{DI}$ in the $\mathrm{C} 1$ and $\mathrm{C} 2$ groups (Figure 1). 


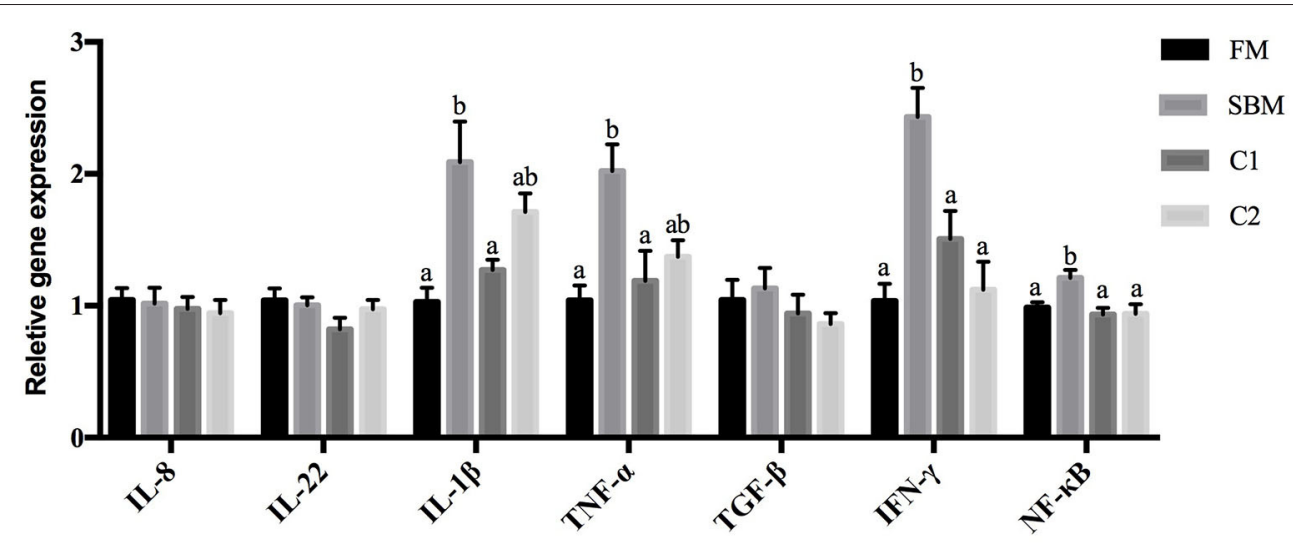

FIGURE 3 | Effects of CAD supplementations on the gene expression level of inflammatory cytokines in the DI of juvenile turbot. Data were expressed as means \pm standard errors of means $(n=9)$. Bars not sharing the same letters denote significant $(P<0.05)$ differences in gene expression level.

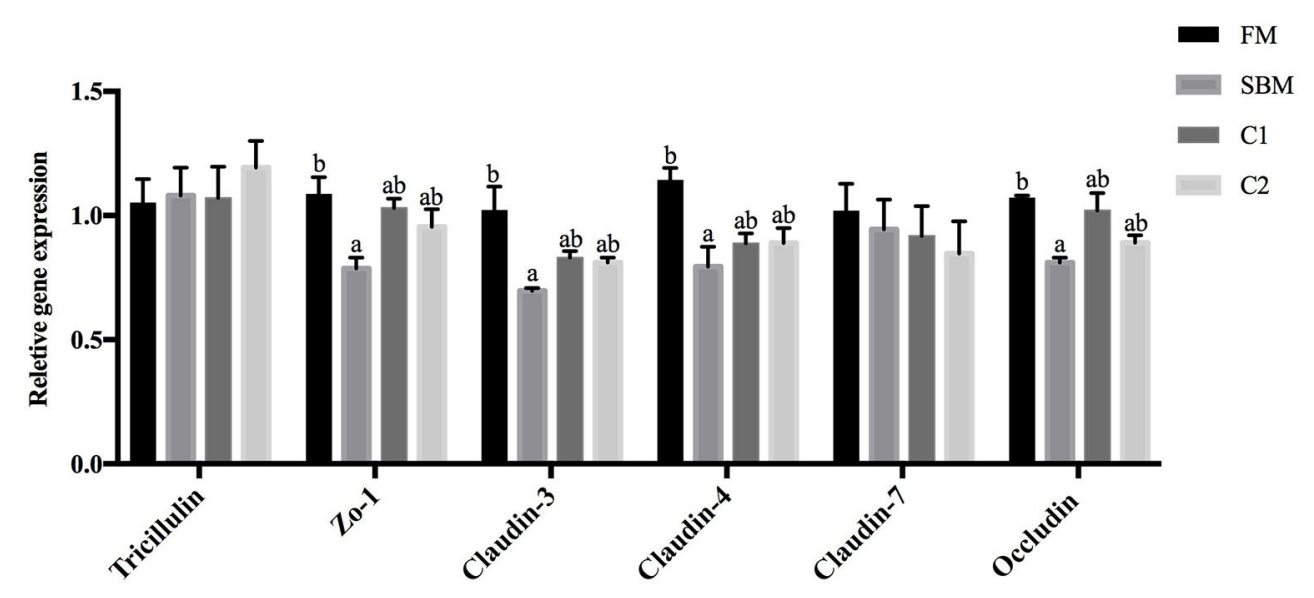

FIGURE 4 | Effects of CAD supplementations on the gene expression level of tight junction protein in the DI of juvenile turbot. Data were expressed as means \pm standard errors of means $(n=9)$. Bars not sharing the same letters denote significant $(P<0.05)$ differences in gene expression level.

\section{Quantitative RT-PCR Analysis of Inflammatory cytokines in DI}

Compared with the FM group, the SBM diet significantly increased gene expression levels of $n f-\kappa b$ p65, if $n-\gamma, \operatorname{tnf}-\alpha$, and $i l 1-\beta$ in DI. However, compared with diet SBM, the gene expression level of these pro-inflammatory genes significantly lowered in turbot fed the diet $\mathrm{C} 1(P<0.05)$. The gene expression levels of $n f-\kappa b$ p65 and ifn- $\gamma$ decreased remarkably in the C2 group $(P<0.05)$. No diet-related differences were observed in the gene expression levels of transforming growth factor- $\beta$ (tgf$\beta$ ), interleukin-8 (il-8), and interleukin-22 (il-22) among all diet groups $(P>0.05$; Figure 3$)$.

\section{Quantitative RT-PCR Analysis of Tight Junction Proteins in the DI}

Compared with the FM group, the turbot subjected to SBM treatment showed significantly decreased intestinal gene expression level of occludin, claudin-3, claudin-4, and $z o-1$
$(P<0.05)$. The supplementation of CAD in SBM increased the gene expression level of occludin, claudin-3, claudin-4, and $z o$ 1 compared with the SBM group and exhibited no significant differences with the FM group. There are no remarkable changes in the gene expression level of tricellulin and claudin-7 among all dietary treatments $(P>0.05$; Figure 4).

\section{Intestinal Microbiota Composition}

A total of 973,130 raw single-end reads was generated from 12 samples, resulting in the identification of 5,840 OTUs according to $97 \%$ sequence similarity. The rarefaction curves reached the plateau, indicating adequate sequencing depth for all the samples (Figure 5). At the phylum level, Firmicutes, Bacteroidetes, and Proteobacteria were detected as the predominant bacterial phyla in the DI of turbot from all groups. At the genus level, Mycoplasms, unidentified Clostridiales, Bacteroides, Lactobacillus, Faecalibacterium, Rhodococcus, Subdoligranulum, unidentified Ruminococcaceae, unidentified Cyanobacteria, and Bacillus composed the top ten dominant genera of intestinal microbiota 
communities (Figure 6). Bacteria diversity (Shannon index) did not differ among all treatments. However, fish in the SBM group showed a trend toward higher bacterial richness (observed species) compared with fish in the FM group, while dietary $\mathrm{CAD}$ decreased the number of observed species compared with the SBM group (Figure 7). Unweighted unifrac-based principal coordinate analysis (PCoA) showed evident clustering of intestinal microbiota samples according to different basic diet composition. FM and SBM groups differed significantly in principal coordinate $1(\mathrm{PC} 1)(P=0.015)$, while the intestinal microbiota of SBM, C1, and C2 was not significantly different from each other (Figure 8). As revealed by LEfSe analysis, the relative abundance of Firmicutes and Blautia were significantly higher in group FM compared with the SBM group (Figure 9). Turbot fed $\mathrm{C} 1$ diet significantly decreased the abundance of Proteobacteria and increased Firmicutes/Bacteroidetes ratio compared with the SBM diet $(P=0.05$; Figures 9, 10).

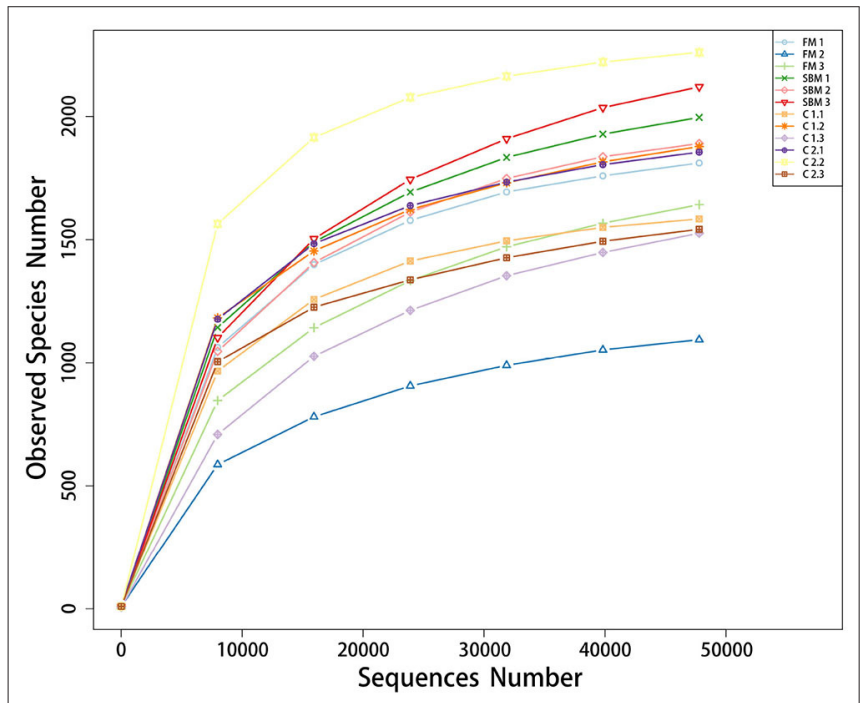

FIGURE 5 | Rarefied curves for the number of observed species in the DI of turbot.

\section{DISCUSSION}

The present study showed that dietary CAD markedly alleviated the symptom of the soybean meal-induced enteritis (SBMIE) in turbot, which was evidenced by the decreased eosinophilic granulocytes count in lamina propria and the reduced gene expression of pro-inflammatory cytokines. In line with our study, intake of porcine $\beta$-Defensin 2 attenuated the DSS-induced colitis by inhibiting the production of inflammatory mediators and preserving the integrity of colon in mice (Han et al., 2015), and Cathelicidin-WA was capable of attenuating intestinal inflammation and diarrhea in weaned diarrheal piglets (Yi et al., 2016). Moreover, it is fairly well elucidated that the development of SBMIE in turbot is in connection with the activation of $n f-$ $\kappa b$ (Chen et al., 2018a; Liu et al., 2018), which in multiple ways regulates the production of various inflammatory cytokines via acting as the transcription factor binding sites (Liu et al., 2003; Sahlmann et al., 2013). In this study, the significantly decreased intestinal gene expression level of $n f-\kappa b$ p65 in fish fed CAD diet indicated that CAD probably blocked the $n f-\kappa b$ triggered overexpression of intestinal inflammatory cytokines, which is supported by the evidences that cecropin family peptides efficiently inhibit LPS or pathogens-induced expression level of $n f-\kappa b$ in mouse peritoneal macrophages (Wu et al., 2015; Wei et al., 2018).

The present results concerning the perimeter ratio of intestinal villi suggested that $\mathrm{CAD}$ contributed to a better preserved morphological structure in turbot, implying an improved absorbency of nutrients either by an increased intestinal villi length and/or amount of mucosal folding (Dimitroglou et al., 2009). As an important component of the intestinal epithelium, tight junction (TJ) located most apical mucosa acting as a selective physical barrier to defend against numerous harmful substances (Tsukita et al., 2001). Dietary CAD increased the gene expression level of intestinal claudin-3, claudin-4, zo-1, and occludin in turbot, which is in line with the previous study showing that the Cathelicidin-BF increased mRNA level of $z o-1$, occludin, and claudin-1 in the colon of mice with colitis induced by DSS (Zhang et al., 2015). Likewise, human defensin 5 was able to block the ethyl alcohol and colitisinduced disruption of colonic TJ complexes in mice (Shukla et al.,
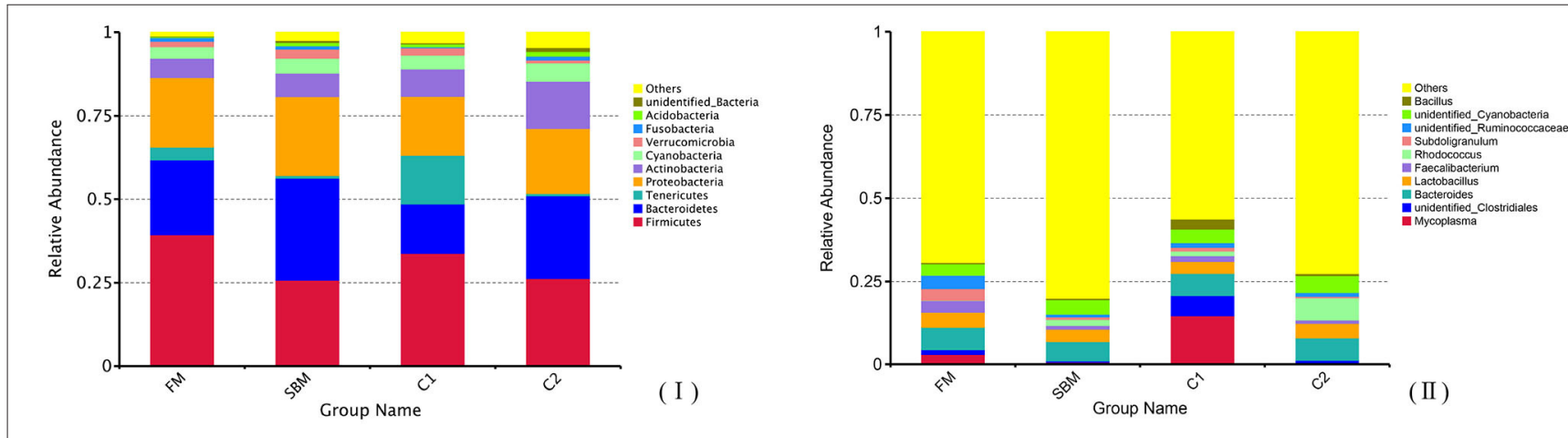

FIGURE 6 | Top 10 most abundant taxa at phylum (I) and genus level (II) in the DI of turbot. 



(II)

FIGURE 7 | $\alpha$-diversity represented by observed species (I) and Shannon diversity (II) in the DI of turbot.

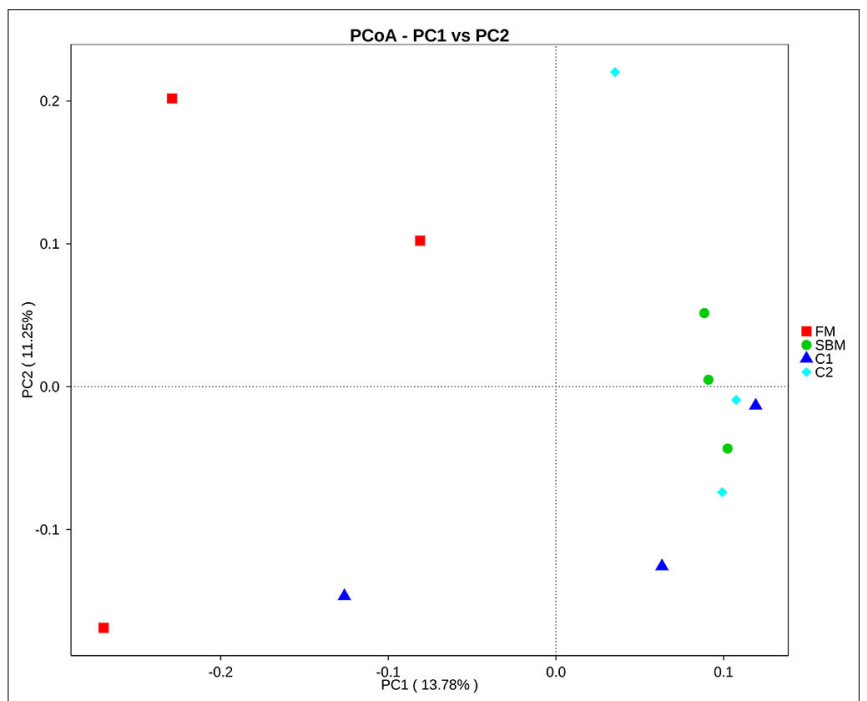

FIGURE 8 | Unweighted unifrac-based principal coordinate analysis (PCoA) analysis in the DI of turbot. Small dots represent individual samples.

2018). Since the destroy in intestinal TJ function would lead to a paracellular influx of luminal antigens, which is a crucial pathogenic factor in initiation and exacerbation of intestinal inflammation (Groschwitz and Hogan, 2009), it is proposed that the reduced intestinal inflammation in turbot fed dietary CAD may be partly due to the improved of barrier function, although the mechanism remains to be fully defined and understood.

Diets play a key role in shaping intestinal microbiota composition, which in turn well-reflects host intestinal health status (Brown et al., 2012; Pop, 2012). In the present study, the number of observed species in the SBM group was significantly increased compared with the FM group, which was in agreement with the studies in rainbow trout (Oncorhynchus mykiss) (Desai et al., 2012) and Atlantic salmon (Salmo salar) (Anne Marie et al., 2007). However, this number was strongly decreased by dietary CAD compared with the SBM group, which is in accordance with our previous finding that a diet with high supplementation of CAD significantly decreased the $\alpha$-diversity of intestinal microbiota in turbot (Dai et al., 2020). The descended number of observed species clearly reflect the effective antimicrobial property of $\mathrm{CAD}$ in fish intestine just as it has been found to decrease the total aerobic bacterial counts in the jejunal and caecal digesta in broilers (Wen and $\mathrm{He}, 2012$ ) and piglets (Wu et al., 2012). In terms of intestinal microbiota composition, Proteobacteria, Bacteroidetes, and Firmicutes constitute the three major members of bacteria at the phylum level in all treatment groups. This result was in agreement with the previous findings in turbot whose intestinal microbiome was unveiled by bacterial Next-Generation Sequencing (Li et al., 2017; Dai et al., 2018, 2020; Yang et al., 2018). However, a significant decrease in the relative abundance of Firmicutes was observed in SBM group compared with FM group, which was mainly attributed to losses of Blautia, while no such a difference was found between $\mathrm{C} 1$ and FM group, suggesting the relative abundance of Blautia was profitably increased by dietary CAD. Blautia is well-recognized as part of the butyrate-producing bacteria genus of the intestinal microbiota (Biddle et al., 2013; De Filippis et al., 2016), and some of Blautia species were able to reduce the ratio of pro- to anti-inflammatory cytokines ratio in human mononuclear cells (Benítez-Páez et al., 2020). Therefore, it is possible that the thriving of Blautia by diet 


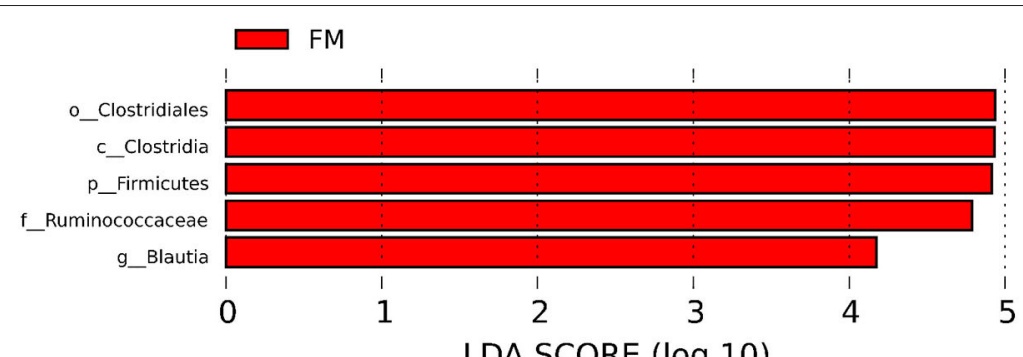

LDA SCORE ( $\log 10)$

( I )

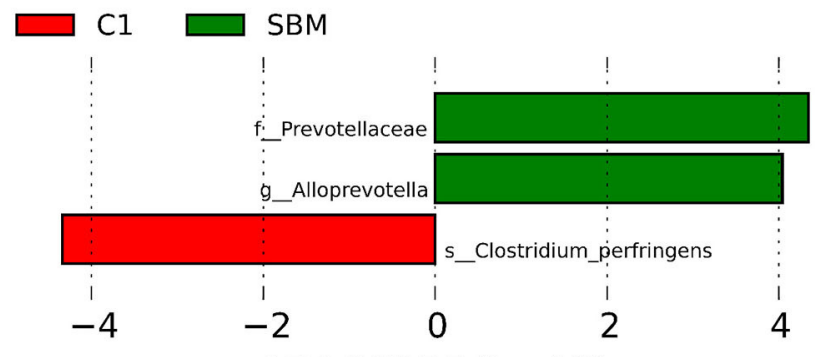

LDA SCORE ( $\log 10)$

( II )

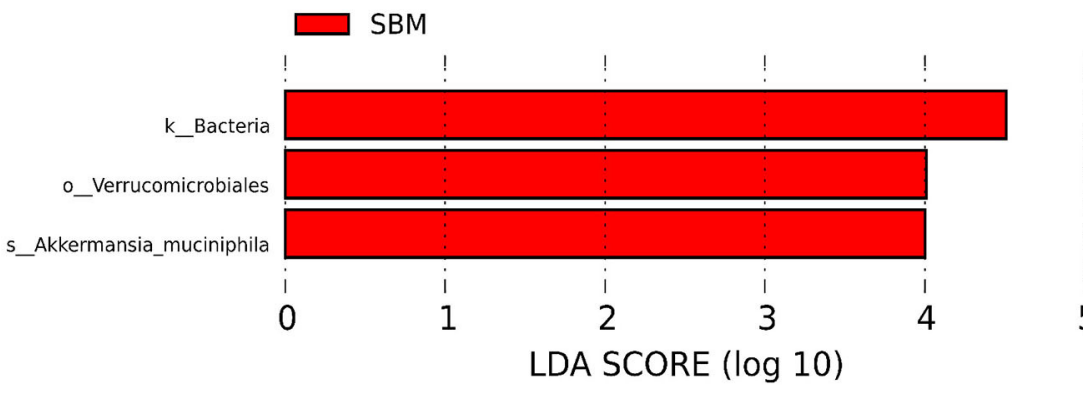

(III)

FIGURE 9 | The Linear Discriminant Analysis (LDA score >4) of microbiota communities in the DI of turbot. (I) FM vs. SBM; (II) SBM vs. C1; (III) SBM vs. C2.

C1 might facilitate the inflammation regulation. Besides, diet C1 markedly increased Firmicutes/Bacteroidetes ratio, which is an indicator closely associated with human intestinal health, significantly decreasing among people with various gut disorders such as irritable bowel syndrome and inflammatory bowel diseases (Tamboli et al., 2004; Collins, 2014). Additionally, there is an obvious reduction in members of family Prevotellaceae in the C1 group compared with the SBM group. Prevotellaceae has been reported as a prominent representation in samples from IBD patients (Kleessen et al., 2002; Lucke et al., 2006), which may disrupt the mucosal barrier function by producing sulfatases that actively degrade mucus oligosaccharide (Wright et al., 2000). Taken together, these results point to a bacterial component as being responsible for an inflammatory phenotype in SBMIE in turbot, while the shaping of microbiota composition by dietary CAD probably posed a positive effect on intestinal inflammation status.

Collectively, diet with CAD supplementation could effectively mitigate the intestinal enteritis of turbot induced by SBM, which was evidenced by inhibiting the overexpression of intestinal pro-inflammatory cytokines, enhancing the intestinal mucosal

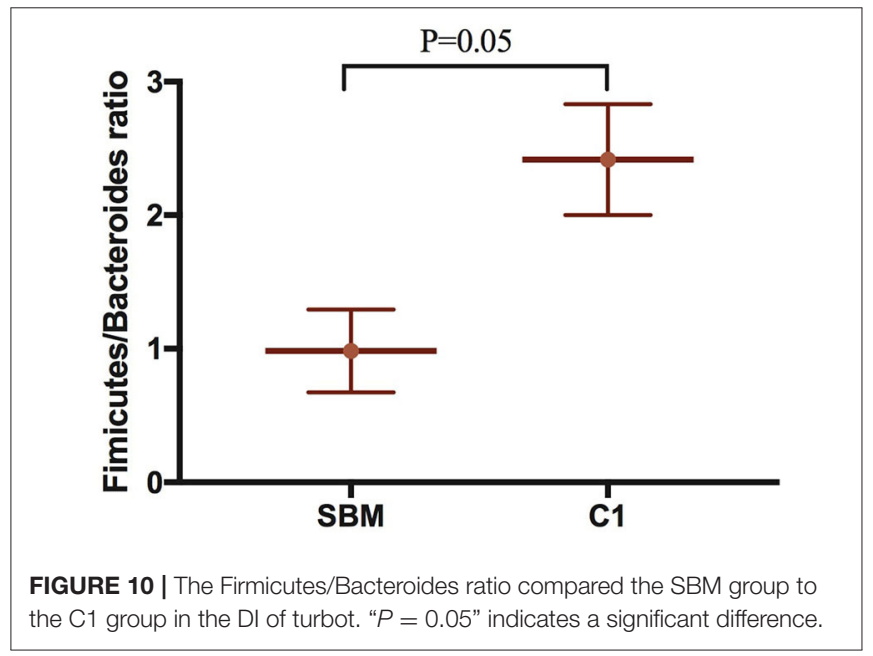

barriers, as well as improving the intestinal microbiota profiles. The improved intestinal health eventually provides the increased feed efficiency and a better growth performance of turbot. 
This study indicated that CAD could serve as an effective additive to promote intestinal health in turbot fed high levels of soybean meal.

\section{DATA AVAILABILITY STATEMENT}

The datasets presented in this study can be found in online repositories. The names of the repository/repositories and accession number(s) can be found below: https://bigd.big.ac. cn/, CRA002769.

\section{ETHICS STATEMENT}

The animal study was reviewed and approved by Animal Care and Use Committee of Ocean University of China.

\section{REFERENCES}

Anne Marie, B. M., Penn, M. H., Patricia Mora, S., Ståle, R., Sigmund, S., Thor, L., et al. (2007). Effects of dietary soyabean meal, inulin and oxytetracycline on intestinal microbiota and epithelial cell stress, apoptosis and proliferation in the teleost Atlantic salmon (Salmo salar L.). Br. J. Nutr. 97, 699-713. doi: $10.1017 /$ S0007114507381397

AOAC (1995). Official Methods of Analysis of Association of Official Analytical Chemists International. Arlington, VA: AOAC.

Baeverfjord, G., and Krogdahl, A. (1996). Development and regression of soybean meal induced enteritis in Atlantic salmon, Salmo salar L., distal intestine: a comparison with the intestines of fasted fish. J. Fish Dis. 19, 375-387. doi: 10.1046/j.1365-2761.1996.d01-92.x

Benítez-Páez, A., del Pugar, E. M. G., López-Almela, I., Moya-Pérez, Á., CodoñerFranch, P., and Sanz, Y. (2020). Depletion of Blautia species in the microbiota of obese children eelates to intestinal inflammation and metabolic phenotype worsening. mSystems 5:e0587-19. doi: 10.1128/mSystems.00857-19

Biddle, A., Stewart, L., Blanchard, J., and Leschine, S. (2013). Untangling the genetic basis of fibrolytic specialization by Lachnospiraceae and Ruminococcaceae in diverse gut communities. Diversity 5, 627-640. doi: $10.3390 / \mathrm{d} 5030627$

Brown, K., DeCoffe, D., Molcan, E., and Gibson, D. L. (2012). Diet-induced dysbiosis of the intestinal microbiota and the effects on immunity and disease. Nutrients 4, 1095-1119. doi: 10.3390/nu4081095

Chen, X., Zhu, F., Cao, Y., and Qiao, S. (2009). Novel expression vector for secretion of cecropin $\mathrm{AD}$ in Bacillus subtilis with enhanced antimicrobial activity. Antimicrob. Agents Chemother. 53, 3683-3689. doi: 10.1128/AAC.00251-09

Chen, Z., Liu, Y., Li, Y., Yang, P., Hu, H., Yu, G., et al. (2018a). Dietary arginine supplementation mitigates the soybean meal induced enteropathy in juvenile turbot, Scophthalmus maximus L. Aquac. Res. 49, 1535-1545. doi: 10.1111 /are. 13608

Chen, Z., Zhao, S., Liu, Y., Yang, P., Ai, Q., Zhang, W., et al. (2018b). Dietary citric acid supplementation alleviates soybean meal-induced intestinal oxidative damage and micro-ecological imbalance in juvenile turbot, Scophthalmus maximus L. Aquac. Res. 49, 3804-3816. doi: 10.1111/are.13847

Clements, K. D., Angert, E. R., Montgomery, W. L., and Choat, J. H. (2014). Intestinal microbiota in fishes: what's known and what's not. Mol. Ecol. 23, 1891-1898. doi: 10.1111/mec.12699

Collins, S. M. (2014). A role for the gut microbiota in IBS. Nat. Rev. Gastroenterol. Hepatol. 11, 497-505. doi: 10.1038/nrgastro.2014.40

Dai, J., Li, Y., Yang, P., Liu, Y., Chen, Z., Ou, W., et al. (2018). Citric acid as a functional supplement in diets for juvenile turbot, Scophthalmus maximus L.: effects on phosphorus discharge, growth performance, and intestinal health. Aquaculture 495, 643-653. doi: 10.1016/j.aquaculture.2018.04.004

Dai, J., Zheng, J., Ou, W., Xu, W., Ai, Q., Zhang, W., et al. (2020). The effect of dietary cecropin $\mathrm{AD}$ on intestinal health, immune response and disease resistance of juvenile turbot (Scophthalmus maximus L.). Fish Shellfish Immunol. 100, 117-125. doi: 10.1016/j.fsi.2020.02.052

\section{AUTHOR CONTRIBUTIONS}

YZ and JD designed the experiments. JD, WO, and GY contributed to performing the experiment and the data analysis. JD and YZ wrote the paper. YZ, QA, WZ, and KM contributed to revise the manuscript. All authors contributed to the article and approved the submitted version.

\section{FUNDING}

This research was financially supported by National Key R\&D Program of China (Grant No. 2019YFD0900104), National Natural Science Foundation of China (Grant No. 31872577 and 41576137), and China Agriculture Researches System (Grant No. CARS 47-G10).

De Filippis, F., Pellegrini, N., Vannini, L., Jeffery, I. B., La Storia, A., Laghi, L., et al. (2016). High-level adherence to a Mediterranean diet beneficially impacts the gut microbiota and associated metabolome. Gut 65, 1812-1821. doi: 10.1136/gutjnl-2015-309957

DeMeo, M. T., Mutlu, E. A., Keshavarzian, A., and Tobin, M. C. (2002). Intestinal permeation and gastrointestinal disease. J. Clin. Gastroenterol. 34, 385-396. doi: 10.1097/00004836-200204000-00003

Desai, A. R., Links, M. G., Collins, S. A., Mansfield, G. S., Drew, M. D., Van Kessel, A. G., et al. (2012). Effects of plant-based diets on the distal gut microbiome of rainbow trout (Oncorhynchus mykiss). Aquaculture 350, 134-142. doi: 10.1016/j.aquaculture.2012.04.005

Dimitroglou, A., Merrifield, D., Moate, R., Davies, S., Spring, P., Sweetman, J., et al. (2009). Dietary mannan oligosaccharide supplementation modulates intestinal microbial ecology and improves gut morphology of rainbow trout, Oncorhynchus mykiss (Walbaum). J. Anim. Sci. 87, 3226-3234. doi: $10.2527 /$ jas.2008-1428

Dong, X.-Q., Zhang, D.-M., Chen, Y.-K., Wang, Q.-J., and Yang, Y.-Y. (2015). Effects of antimicrobial peptides (AMPs) on blood biochemical parameters, antioxidase activity, and immune function in the common carp (Cyprinus carpio). Fish Shellfish Immunol. 47, 429-434. doi: 10.1016/j.fsi.2015. 09.030

Groschwitz, K. R., and Hogan, S. P. (2009). Intestinal barrier function: molecular regulation and disease pathogenesis. J. Allergy Clin. Immunol. 124, 3-20. doi: $10.1016 /$ j.jaci.2009.05.038

Han, F., Zhang, H., Xia, X., Xiong, H., Song, D., Zong, X., et al. (2015). Porcine $\beta$-defensin 2 attenuates inflammation and mucosal lesions in dextran sodium sulfate-induced colitis. J. Immunol. 194, 1882-1893. doi: 10.4049/jimmunol.1402300

Hancock, R. E. W., Haney, E. F., and Gill, E. E. (2016). The immunology of host defence peptides: beyond antimicrobial activity. Nat. Rev. Immunol. 16, 321-334. doi: 10.1038/nri.2016.29

Kim, J.-K., Lee, E., Shin, S., Jeong, K.-W., Lee, J.-Y., Bae, S.-Y., et al. (2011) Structure and function of papiliocin with antimicrobial and anti-inflammatory activities isolated from the swallowtail butterfly, Papilio xuthus. J. Biol. Chem. 286, 41296-41311. doi: 10.1074/jbc.M111.269225

Kleessen, B., Kroesen, A., Buhr, H., and Blaut, M. (2002). Mucosal and invading bacteria in patients with inflammatory bowel disease compared with controls. Scand. J. Gastroenterol. 37, 1034-1041. doi: 10.1080/003655202320378220

Krogdahl, A., Bakke-McKellep, A., and Baeverfjord, G. (2003). Effects of graded levels of standard soybean meal on intestinal structure, mucosal enzyme activities, and pancreatic response in Atlantic salmon (Salmo salar L.). Aquac. Nutr. 9, 361-371. doi: 10.1046/j.1365-2095.2003.00264.x

Lee, E., Shin, A., and Kim, Y. (2015). Anti-inflammatory activities of cecropin $\mathrm{a}$ and its mechanism of action. Arch. Insect Biochem. Physiol. 88, 31-44. doi: 10.1002/arch. 21193

Li, S., Chi, S., Cheng, X., Wu, C., Xu, Q., Qu, P., et al. (2020). Effects of antimicrobial peptides on the growth performance, antioxidant and intestinal function in juvenile largemouth bass, Micropterus salmoides. Aquac. Rep. 16:100252. doi: 10.1016/j.aqrep.2019.100252 
Li, Y., Yang, P., Zhang, Y., Ai, Q., Xu, W., Zhang, W., et al. (2017). Effects of dietary glycinin on the growth performance, digestion, intestinal morphology and bacterial community of juvenile turbot, Scophthalmus maximus L. Aquaculture 479, 125-133. doi: 10.1016/j.aquaculture.2017.05.008

Lin, X., Chen, W., Lin, S., and Luo, L. (2015). Effects of dietary cecropin on growth, non-specific immunity and disease resistance of tilapia (O. reochromis niloticus $\times$ O. aureus). Aquac. Res. 46, 2999-3007. doi: 10.1111/are.12457

Liu, H., Pan, C., Liu, Q., Yang, W., and Liu, X. (2003). Effect of NF-кB and p38 MAPK in activated monocytes/macrophages on pro-inflammatory cytokines of rats with acute pancreatitis. World J. Gastroenterol. 9, 2513-2518. doi: 10.3748/wjg.v9.i11.2513

Liu, Y., Chen, Z., Dai, J., Yang, P., and Mai, K. (2018). The protective role of glutamine on enteropathy induced by high dose of soybean meal in turbot, Scophthalmus maximus L. Aquaculture 497, 510-519. doi: 10.1016/j.aquaculture.2018.08.021

Liu, Y., Chen, Z., Dai, J., Yang, P., Xu, W., Ai, Q., et al. (2019). Sodium butyrate supplementation in high-soybean meal diets for turbot (Scophthalmus maximus L.): effects on inflammatory status, mucosal barriers and microbiota in the intestine. Fish Shellfish Immunol. 88, 65-75. doi: 10.1016/j.fsi.2019.02.064

Lucke, K., Miehlke, S., Jacobs, E., and Schuppler, M. (2006). Prevalence of Bacteroides and Prevotella spp. in ulcerative colitis. J. Med. Microbiol. 55, 617-624. doi: 10.1099/jmm.0.46198-0

Luis Balcázar, J., Decamp, O., Vendrell, D., De Blas, I., and Ruiz-Zarzuela, I. (2006). Health and nutritional properties of probiotics in fish and shellfish. Microb. Ecol. Health Dis. 18, 65-70. doi: 10.1080/08910600600799497

Mansfield, G. S., Desai, A. R., Nilson, S. A., Van Kessel, A. G., Drew, M. D., and Hill, J. E. (2010). Characterization of rainbow trout (Oncorhynchus mykiss) intestinal microbiota and inflammatory marker gene expression in a recirculating aquaculture system. Aquaculture 307, 95-104. doi: 10.1016/j.aquaculture.2010.07.014

Merrifield, D. L., Dimitroglou, A., Bradley, G., Baker, R. T. M., and Davies, S. J. (2009). Soybean meal alters autochthonous microbial populations, microvilli morphology and compromises intestinal enterocyte integrity of rainbow trout, Oncorhynchus mykiss (Walbaum). J. Fish Dis. 32, 755-766. doi: 10.1111/j.1365-2761.2009.01052.x

Pop, M. (2012). We are what we eat: how the diet of infants affects their gut microbiome. Genome Biol. 13:152. doi: 10.1186/gb-2012-13-4-152

Reddy, K. V. R., Yedery, R. D., and Aranha, C. (2004). Antimicrobial peptides: premises and promises. Int. J. Antimicrob. Agents 24, 536-547. doi: 10.1016/j.ijantimicag.2004.09.005

Refstie, S., Baeverfjord, G., Seim, R. R., and Elveb,ø, O. (2010). Effects of dietary yeast cell wall $\beta$-glucans and MOS on performance, gut health, and salmon lice resistance in Atlantic salmon (Salmo salar) fed sunflower and soybean meal. Aquaculture 305, 109-116. doi: 10.1016/j.aquaculture.2010.04.005

Reveco, F. E., Øverland, M., Romarheim, O. H., and Mydland, L.T. (2014). Intestinal bacterial community structure differs between healthy and inflamed intestines in Atlantic salmon (Salmo salar L.). Aquaculture 420, 262-269. doi: 10.1016/j.aquaculture.2013.11.007

Sahlmann, C., Sutherland, B. J. G., Kortner, T. M., Koop, B. F., Krogdahl, A., and Bakke, A. M. (2013). Early response of gene expression in the distal intestine of Atlantic salmon (Salmo salar L.) during the development of soybean meal induced enteritis. Fish Shellfish Immunol. 34, 599-609. doi: 10.1016/j.fsi.2012.11.031

Sealey, W. M., Barrows, F. T., Smith, C. E., Overturf, K., and LaPatra, S. E. (2009). Soybean meal level and probiotics in first feeding fry diets alter the ability of rainbow trout Oncorhynchus mykiss to utilize high levels of soybean meal during grow-out. Aquaculture 293, 195-203. doi: 10.1016/j.aquaculture.2009.04.013

Shukla, P. K., Meena, A. S., Rao, V., Rao, R. G., Balazs, L., and Rao, R. (2018). Human defensin-5 blocks ethanol and colitis-induced dysbiosis, tight junction disruption and inflammation in mouse intestine. Sci. Rep 8:16241. doi: 10.1038/s41598-018-34263-4

Su, L., Nalle, S. C., Shen, L., Turner, E. S., Singh, G., Breskin, L. A., et al. (2013). TNFR2 activates MLCK-dependent tight junction dysregulation to cause apoptosis-mediated barrier loss and experimental colitis. Gastroenterology 145, 407-415. doi: 10.1053/j.gastro.2013.04.011

Su, Y.-L., Chen, G., Chen, L.-S., Li, J.-Z., Wang, G., He, J.-Y., et al. (2019). Effects of antimicrobial peptides on serum biochemical parameters, antioxidant activity and non-specific immune responses in Epinephelus coioides. Fish Shellfish Immunol. 86, 1081-1087. doi: 10.1016/j.fsi.2018.12.056

Tamboli, C. P., Neut, C., Desreumaux, P., and Colombel, J. (2004). Dysbiosis in inflammatory bowel disease. Gut 53, 1-4. doi: 10.1136/gut.53.1.1

Tsukita, S., Furuse, M., and Itoh, M. (2001). Multifunctional strands in tight junctions. Nat. Rev. Mol. Cell Biol. 2, 285-293. doi: 10.1038/35067088

Ulloa, P. E., Solís, C. J., Paz, J. F. D. L., Alaurent, T. G. S., Caruffo, M., Hernández, A. J., et al. (2016). Lactoferrin decreases the intestinal inflammation triggered by a soybean meal-based diet in zebrafish. J. Immunol. Res. 2016:1639720. doi: 10.1155/2016/1639720

Wei, L., Yang, Y., Zhou, Y., Li, M., Yang, H., Mu, L., et al. (2018). Anti-inflammatory activities of Aedes aegypti cecropins and their protection against murine endotoxin shock. Parasit. Vectors 11:470. doi: 10.1186/s13071-018-3000-8

Wen, L. F., and He, J. G. (2012). Dose-response effects of an antimicrobial peptide, a cecropin hybrid, on growth performance, nutrient utilisation, bacterial counts in the digesta and intestinal morphology in broilers. Br. J. Nutr. 108, 1756-1763. doi: 10.1017/S0007114511007240

Wright, D. P., Rosendale, D. I., and Roberton, A. M. (2000). Prevotella enzymes involved in mucin oligosaccharide degradation and evidence for a small operon of genes expressed during growth on mucin. FEMS Microbiol. Lett. 190, 73-79. doi: 10.1111/j.1574-6968.2000.tb09265.x

Wu, J., Mu, L., Zhuang, L., Han, Y., Liu, T., Li, J., et al. (2015). A cecropinlike antimicrobial peptide with anti-inflammatory activity from the black fly salivary glands. Parasit. Vectors 8:561. doi: 10.1186/s13071-015-1176-8

Wu, S., Zhang, F., Huang, Z., Liu, H., Xie, C., Zhang, J., et al. (2012). Effects of the antimicrobial peptide cecropin $\mathrm{AD}$ on performance and intestinal health in weaned piglets challenged with Escherichia coli. Peptides 35, 225-230. doi: 10.1016/j.peptides.2012.03.030

Yang, P., Hu, H., Liu, Y., Li, Y., Ai, Q., Xu, W., et al. (2018). Dietary stachyose altered the intestinal microbiota profile and improved the intestinal mucosal barrier function of juvenile turbot, Scophthalmus maximus L. Aquaculture 486, 98-106. doi: 10.1016/j.aquaculture.2017.12.014

Yi, H., Zhang, L., Gan, Z., Xiong, H., Yu, C., Du, H., et al. (2016). High therapeutic efficacy of cathelicidin-WA against postweaning diarrhea via inhibiting inflammation and enhancing epithelial barrier in the intestine. Sci. Rep. 6:25679. doi: 10.1038/srep25679

Zhai, Z., Ni, X., Jin, C., Ren, W., Li, J., Deng, J., et al. (2018). Cecropin a modulates tight junction-related protein expression and enhances the barrier function of porcine intestine epithelial cells by suppressing the MEK/ERK pathway. Int. J. Mol. Sci. 19:1941. doi: 10.3390/ijms19071941

Zhang, H., Xia, X., Han, F., Jiang, Q., Rong, Y., Song, D., et al. (2015). CathelicidinBF, a novel antimicrobial peptide from Bungarus fasciatus, attenuates disease in a dextran sulfate sodium model of colitis. Mol. Pharm. 12, 1648-1661. doi: 10.1021/acs.molpharmaceut.5b00069

Zhao, S., Chen, Z., Zheng, J., Dai, J., Ou, W., Xu, W., et al. (2019). Citric acid mitigates soybean meal induced inflammatory response and tight junction disruption by altering TLR signal transduction in the intestine of turbot, Scophthalmus maximus L. Fish Shellfish Immunol. 92, 181-187. doi: 10.1016/j.fsi.2019.06.004

Zhou, X. X., Wang, Y. B., and Li, W. F. (2008). Effect of feeding apidaecin on common carp (Cyprinus carpio) growth performances and immune function. Aquaculture 279, 108-112. doi: 10.1016/j.aquaculture.2008.04.024

Zhou, Z., Ringo, E., Olsen, R. E., and Song, S. K. (2018). Dietary effects of soybean products on gut microbiota and immunity of aquatic animals: a review. Aquac. Nutr. 24, 644-665. doi: 10.1111/anu. 12532

Conflict of Interest: The authors declare that the research was conducted in the absence of any commercial or financial relationships that could be construed as a potential conflict of interest.

Copyright (c) $2020 \mathrm{Dai}, \mathrm{Ou}, \mathrm{Yu}, \mathrm{Ai}$, Zhang, Mai and Zhang. This is an open-access article distributed under the terms of the Creative Commons Attribution License (CC BY). The use, distribution or reproduction in other forums is permitted, provided the original author(s) and the copyright owner(s) are credited and that the original publication in this journal is cited, in accordance with accepted academic practice. No use, distribution or reproduction is permitted which does not comply with these terms. 